\title{
La smart mobility nell'ordinamento giuridico dell'UE e le potenzialità di sviluppo nelle città europee
}

\author{
Christoph Perathoner
}

\section{Verso una nuova concezione di trasporto e mobilità: il fenomeno smart - ideologia e progresso}

L'emergenza epidemiologica da COVID-19, ${ }^{1}$ che, partendo dalla Cina, in poche settimane si è propagata in tutti i continenti di questo pianeta, mettendone in ginocchio la popolazione, ha legittimato i governi ${ }^{2}$ di molti Paesi ad emanare provvedimenti restrittivi, conferendo di fatto agli Stati il potere di comprimere le libertà e i diritti fondamentali ${ }^{3}$ dei singoli in maniera del tutto inedita per le nostre generazioni.

\footnotetext{
${ }^{1}$ Con Dichiarazione dell'Organizzazione Mondiale della Sanità (OMS) del 30 gennaio 2020 l'epidemia da COVID-19 è stata valutata come un'emergenza di sanità pubblica di rilevanza internazionale. Con successiva Dichiarazione dell'Organizzazione Mondiale della Sanità dell'11 marzo 2020 con la quale l'epidemia da COVID-19 è stata valutata come "pandemia" in considerazione dei livelli di diffusività e gravità raggiunti a livello globale, v. online: https://www.who.int/health-topics/coronavirus\#tab=tab_1 (30.05.2020).

${ }^{2}$ In Italia con Delibera del Consiglio dei Ministri 31 gennaio 2020: Dichiarazione dello stato di emergenza in conseguenza del rischio sanitario connesso all'insorgenza di patologie derivanti da agenti virali trasmissibili, in: Gazzetta Ufficiale, $1^{\circ}$ febbraio 2020, n. 26, è stato dichiarato per sei mesi lo stato di emergenza sul territorio nazionale relativo al rischio sanitario da virus COVID-19. Per le misure adottate dal Governo italiano in materia di Coronavirus v. online: http://www.governo.it/it/coronavirus-misure-del-governo (30.05.2020).

${ }^{3} \mathrm{Cfr}$. Beniamino Caravita, L'Italia ai tempi del coronavirus: rileggendo la Costituzione italiana, in: federalismi.it, editoriale - 18 marzo 2020, pp. 3 ss., online: https://www.federalismi.it/nv14/editoriale.cfm?eid=548 (30.05.2020); Gian Luigi Gatta, Coronavirus, limitazione di diritti e libertà fondamentali, e diritto penale: un deficit di legalità da rimediare, in: Sistemapenale.it, Opinioni, 16 marzo 2020, online: https://sistemapenale.it/it/opinioni/coronavisus-covid-19-diritti-liberta-fondamentali-diritto-penale-legalita?out=print (30.05.2020).
}

\section{Perathoner $(\bowtie)$}

Rechtsanwaltssozietät Christoph Perathoner \& Partner, Bozen, Italien

E-Mail: christoph.perathoner@perathoner-partner.com

S. Laimer, C. Perathoner (Hrsg.), Mobilitäts- und Transportrecht in Europa,

Bibliothek des Wirtschaftsrechts 2,

https://doi.org/10.1007/978-3-662-63635-0_6 
Ciò che oggi stiamo vivendo ci costringe a confrontarci con una forma di globalizzazione totalmente nuova, vale a dire la pandemia. Nonostante tale fenomeno certo non positivo - ci renda estremamente fragili, colmandoci di incertezze, possiamo comunque osservare, non senza fiducia, come la scienza medica stia lavorando, in un'ottica di cooperazione globale, ${ }^{4}$ al fine di trovare le cure adeguate per debellare il virus.

La suesposta considerazione vale a mettere in luce quanto il complesso processo di globalizzazione racchiuda in sé indubbiamente grandi potenzialità per lo sviluppo dell'intera umanità, ma al tempo stesso si presenti oggi più che mai disseminato di incognite.

Basti pensare all'inquinamento ambientale, ${ }^{5}$ al cambiamento climatico, alla distruzione delle foreste fluviali tropicali, all'invasione da parte dell'uomo di tutti gli ecosistemi del pianeta, con il conseguente inserimento artificiale di flora e fauna aliene per a tale ecosistema, o all'imminente esaurimento di importanti risorse naturali: tali problematiche globali, al pari del COVID-19, potrebbero sfociare in pericoli oggi ancora non calcolabili per tutta l'umanità.

A ciò si affiancano una crescita della popolazione mondiale esponenziale rispetto agli ultimi millenni ${ }^{6}$ e un aumento del fenomeno migratorio, che ha raggiunto gli apici ed è in continuo aumento ${ }^{7}$ parimenti cresce la percentuale delle persone che scelgono di vivere nelle grandi città, con la conseguenza che si assisterà ad una proliferazione di megalopoli soprattutto in paesi quali India, Cina, Nigeria e non solo. ${ }^{8}$

Questi pochi esempi paiono sintomatici per definire il tempo che stiamo vivendo un periodo di transizione, durante il quale si rende necessario trovare nuove regole che favoriscano l'evoluzione dell'umanità, secondo la logica del progresso, paralizzando l'effetto collaterale dell' autodistruzione, che nessuno può volere; anzi, il progresso dovrebbe essere proprio l'opposto di questo. Il padre della sociologia tedesca Ferdinand Tönnies (1855-1936), nella sua opera Fortschritt und soziale

${ }^{4}$ V. UNGA Res 74/270, 2 aprile 2020: Global solidarity to fight the coronavirus disease 2019 (COVID-19).

${ }^{5}$ All' Assemblea delle Nazioni Unite per l'ambiente (UNEA) di Nairobi, tenutosi dall'11 al 15 marzo 2019, è stato rilevato il dato sconcertante che un quarto delle morti premature è dovuto all'inquinamento e che molti ecosistemi sono vicini alla crisi globale, v. Fourth session of the UN Environment Assembly of the UN Environment Programme (UNEP) 11-15 March 2019, United Nations Environment Programme Headquarters, Nairobi, Kenya, online: https://web.unep.org/environmentassembly/documents/fourth-session-un-environment-assembly (30.05.2020).

${ }^{6}$ Secondo gli ultimi dati pubblicati nel 2019 dal Dipartimento per gli affari economici e sociali delle Nazioni Unite la popolazione mondiale oggi ammonta a 7,7 miliardi di persone. Ci si aspetta che la popolazione mondiale accresca di altri $2 \mathrm{mrd}$. di persone fino al 2050; v. https://population. un.org/wpp/Publications/Files/WPP2019_Highlights.pdf (30.05.2020).

${ }^{7}$ Secondo gli ultimi dati pubblicati nel 2019 dal Dipartimento per gli affari economici e sociali delle Nazioni Unite il numero dei migranti è salito a 272 milioni a livello mondiale (2000: 174 mio.; 2010: 221 mio.); v. https://www.un.org/en/development/desa/population/migration/publications/wallchart/docs/MigrationStock2019_Wallchart.pdf (30.05.2020).

${ }^{8} \mathrm{Cfr}$. https://population.un.org/wup/ (30.05.2020) alla voce "World urbanisation prospects" e v. anche https://www.unfpa.org/urbanization (30.05.2020). 
Entwicklung, ${ }^{9}$ scrisse che il progresso dell'umanità altro non è se non il crescente superamento di carenze o carestia (Mangelzustände).

Orbene, in un mondo urbanizzato, le città del futuro, densamente popolate, sono fortemente esposte a creare condizioni di vita al di sotto degli standard di benessere. Il vero progresso consisterà pertanto nel concepire un nuovo modello di città, che coinvolga tutti i cittadini, che sia in grado di gestire intelligentemente e con risparmio le risorse, che sia economicamente sostenibile, che tuteli l'ambiente, che miri ad essere energeticamente autosufficiente, che sia attenta alla qualità della vita e ai bisogni dei propri cittadini e che sviluppi nuovi modelli per la mobilità urbana ed extraurbana.

Per molti visionari siamo alla soglia di una nuova era, ${ }^{10}$ dove tutto questo dovrebbe essere raggiunto attraverso lo sviluppo delle smart cities $^{11}$ (anche smart regions $^{12}$ ), ovvero di città che sfruttano le nuove tecnologie ${ }^{13}$ nell'ambito della comunicazione e digitalizzazione per connettere telematicamente tutte le infrastrutture materiali di una città con il capitale umano, intellettuale e sociale dei propri abitanti, chiamati anche smart citizens, al fine di trasformare in miglio la qualità di vita e di lavoro, ${ }^{14}$ la sicurezza, le misure ecologico-ambientali, il risparmio energetico e coinvolgendo la maggior parte delle persone possibile. Si tratta pertanto di un modello di città a valenza globale che offre soluzioni idonee - o indispensabili - per risolvere problemi in tutte le città del futuro. Del resto, le tematiche poste a fondamento della concezione di smart city, come la tutela dell'ambiente, l'autosufficienza energetica, il risparmio delle risorse ecc., dovranno prima o poi essere disciplinate da regole comuni per tutti gli Stati.

Oggi viene abusato del concetto di "smart", o meglio "smartness" di modo che, in relazione al tema affrontato, pare più opportuno positivizzare e dire che si tratta di un concetto che va molto di moda: invero, si parla di smart economy, smart people e smart citizens, smart working, smart governance, smart environment, smart living ecc. Tradurre la parola inglese smart in italiano con "intelligente" o "furbo" pare molto riduttivo, in quanto negli ultimi anni il termine smart è stato caricato di tutta una serie di connotati relativi a tecnologia, benessere, sostenibilità ecc., che

\footnotetext{
${ }^{9}$ Cfr. Ferdinand Tönnies, Fortschritt und soziale Entwicklung. Geschichtsphilosophische Ansichten, Karlsruhe 1926, pp. 5 e 36 ss.

${ }^{10} \mathrm{Cfr}$. Nicos Komninos, The Age of Intelligent Cities: Smart Environments and Innovation-for-all strategies, New York-London, 2015.

${ }^{11}$ Ital. città intelligente. Per un approfondimento generale: Edorardo Ferrero, Le smart cities nell'ordinamento giuridico; Sandra Antoniazzi, Smart City; diritto, competenze e obiettivi (realizzabili?) di innovazione, in: federalismi.it, 22 maggio 2019, online: https://www.federalismi.it/ nv14/articolo-documento.cfm?Artid=38648 (30.05.2020).

${ }^{12} \mathrm{Cfr}$. Jorge Marx Gómez/Andreas Solsbach/Thomas Klenke/Volker Wohlgemuth (a cura di), Smart Cities/Smart Regions - Technische, wirtschaftliche und gesellschaftliche Innovationen (Tagungsband zu den 10 BUIS-Tagen), Wiesbaden, 2019.

${ }^{13} \mathrm{Cfr}$. Oliver Gassmann/Jonas Böhm/Maximilian Palmié (a cura di), Smart Cities: Introducing Digital Innovation to Cities, Bingley (UK), 2019.

${ }^{14}$ Cfr. Lukas Neckermann, Smart Cities, Smart Mobility: Transforming the Way We Live and Work, Leichestershire, 2017.
} 
non sono ricompresi nel termine "intelligente" del lessico italiano; è pertanto bene mantenere il termine inglese, anche perché utilizzato ormai a livello mondiale, benché non in modo univoco.

Conseguentemente, per smart city è da intendersi un concetto che va ben oltre il semplice programma urbanistico, ponendosi come una vera e propria ideologia del futuro, una rivoluzione finalizzata a far divenire la città moderna un Internet of Things an Services e (IoTS). Ovviamente vi sono svariate teorie su quella che potrebbe essere la città modello del futuro e su come tutto debba funzionare e interagire per raggiungere tale obiettivo; si pensi alla actor-network theory, alla cradle to cradle theory, ai teoremi della green economy, ai nuovi concetti di share economy, come alle visioni di urban commons e urban metabolism, ecc. Alcune di queste teorie fanno ricordare il celebre capolavoro Nineteen Eighty-Four ${ }^{15}$ dello scrittore britannico George Orwell (1903-1950) o per altri aspetti Brave New World ${ }^{16}$ dello scrittore britannico-statunitense Aldous Huxley (1894-1963).

A tal riguardo, si osservi che il filosofo e letterato francese Jean-Paul Sartre (1905-1980) scrisse nel dopoguerra in un saggio che «les idéologies sont liberté quand elles se font, oppression quand elles sont faites». ${ }^{17}$ E se studiosi di molte branche del sapere - ingegneri, architetti, economisti, sociologi per citarne alcuni oggi si occupano con fervore del tema di grande attualità delle smart cities, per noi giuristi la frase di Sartre deve fungere da monito per esigere, con altrettanto ardimento, che nel modello di "città intelligente" trovi comunque spazio l'imprescindibile rispetto delle libertà e dei diritti fondamentali.

Pare dunque d'obbligo mantenere un sano senso critico quando si affronta dal punto di vista scientifico il tema della smart city, in quanto troppe sono ancora le incognite e molteplici sono le ideologie che gravitano intorno alla tematica. Pensare, ad esempio, al modello cinese dei social credits in una social scoring or rating system $^{18}$ può fare paura a tutti i cittadini cresciuti con i valori comuni all'UE. Del resto, ogni abuso di big data ${ }^{19}$ desta una certa inquietudine, anche se sino ad oggi

\footnotetext{
${ }^{15}$ George Orwell, Nineteen Eighty-Four, London, 1949.

${ }^{16}$ Aldous Huxley, Brave New World, London, 1932.

${ }^{17}$ Jean-Paul Sartre, Qu'est-ce que la littérature? in: Id., Situations II, Paris, 1948, p. 193. Traduzione: "Le ideologie sono libertà mentre si vanno facendo, oppressione quando sono fatte." V. anche Laura Verdi, La polis del futuro, 15 ottobre 2015, in: Italiana Institute for the Future Magazin, online: http://www.futurimagazine.it/dossier/la-polis-del-futuro/ (30.05.2020).

${ }^{18}$ Il Sistema di Credito Sociale è un sistema di sorveglianza di massa, che si basa su nuove tecnologie in grado di analizzare i big data raccolti dal Governo cinese sui singoli abitanti della Cina e senza rispetto di norme della privacy, come sono conosciute in Europa. In base ai dati raccolti (per esempio, condizioni economiche e sociali) viene calcolato un punteggio definito "credito sociale" della persona, che è come un "valore", che viene attribuito ad ogni cittadino. In base al credito sociale il Governo premierà o punirà i cittadini. Punizioni possono essere p.e. il divieto di volo e di lasciare il paese, espulsione da scuole, divieto di accedere a determinati mestieri, pubblicazione del proprio nome in liste di demerito; cfr. Mirjam Meissner, China's social credit system. A bigdata enabled approach to market regulation with broad implications for doing business in China, 24 maggio 2017, Mercator Institute for China Studies - Merics, online: https://www.merics.org/ sites/default/files/2017-09/China\%20Monitor_39_SOCS_EN.pdf (30.05.2020).

${ }^{19}$ It. anche megadati; per una nozione del concetto v. Andrea De Mauro/Marco Greco/Michele Gri-
} 
l'UE è stata sempre molto attenta nella tutela dei diritti fondamentali alla protezione dei dati a carattere personale. ${ }^{20}$

Ad ogni modo, nessun modello di smart city può essere concepito se non in correlazione con il settore del trasporto e della mobilità, che congiuntamente all'energia costituiscono la linfa vitale di ogni città del futuro. Come delle vere e proprie arterie e vene nel corpo umano, il trasporto di persone e di merci garantisce la vita di una città.

\section{Il concetto della smart mobility}

Il trasporto e la mobilità delle smart cities vengono definite come smart mobility. Non esiste una definizione olistica accettata universalmente dal mondo accademico, politico, sociale ed economico di quale sia la smart mobility. Tale espressione viene comunque frequentemente utilizzata e diffusa dal mondo politico come slogan visionario, ma viene impiegata anche da imprese operanti nel settore per attirare l'attenzione su propri prodotti o servizi. ${ }^{21}$

La smart mobility vuole implementare le nuove tecnologie della comunicazione, della digitalizzazione e dell'informatica in ogni settore dell'ingegneria dei trasporti per pianificare e progettare meglio il trasporto e la mobilità, ma anche per connettere telematicamente tutte le infrastrutture materiali e per migliorare la manutenzione e la gestione dei sistemi e dei mezzi di trasporto. ${ }^{22}$ Il tutto con lo scopo di

maldi, A formal definition of Big Data based on its essential features, in: Library Review, 2016, vol. 65, n. 3, pp. 122-135, https://doi.org/10.1108/LR-06-2015-0061 (20.05.2020).

${ }^{20} \mathrm{Il}$ diritto alla protezione dei dati a carattere personale è garantito dall'articolo 8 della Carta dei diritti fondamentali dell'Unione europea, dal Regolamento (UE) 2016/679 del Parlamento europeo e del Consiglio, del 27 aprile 2016, relativo alla protezione delle persone fisiche con riguardo al trattamento dei dati personali, nonché alla libera circolazione di tali dati e che abroga la direttiva 95/46/CE, in: GUUE, 4 maggio 2016, L 119, pp. 1 ss., dalla Direttiva 2002/58/CE del Parlamento europeo e del Consiglio, del 12 luglio 2002, relativa al trattamento dei dati personali e alla tutela della vita privata nel settore delle comunicazioni elettroniche, in: GUUE, 31 luglio 2002, L 201, pp. 37 ss. Nel Libro Bianco del 2020 sull Intelligenza Artificiale (IA) viene sottolineata l'esigenza di evitare che si impongano, nella corsa tecnologica, modelli contrari ai principi fondamentali dell'UE; v. Libro Bianco della Commissione europea del 19 febbraio 2020 sull'intelligenza artifici-

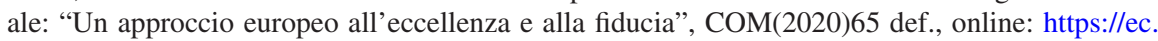
europa.eu/info/sites/info/files/commission-white-paper-artificial-intelligence-feb2020_it.pdf. A p. 10 del Libro Bianco si legge: "La Commissione è convinta che la cooperazione internazionale sulle questioni riguardanti l'IA debba basarsi su un approccio che promuova il rispetto dei diritti fondamentali, tra cui la dignità umana, il pluralismo, l'inclusione, la non discriminazione e la protezione della privacy e dei dati personali26, e si adopererà per esportare $i$ suoi valori nel mondo."

${ }^{21}$ Cfr. p.e. www.smartmobility.com o www.smartmobility.it (30.05.2020).

${ }^{22}$ Per molti aspetti pratici v. Barbara Flügge (a cura di), Smart Mobility: Trends, Konzepte, Best Practices für die intelligente Mobilität, Wiesbaden 2016; Barbara Flügge (a cura di), Smart Mobility - Connecting Everyone: Trends, Concepts and Best Practices, Wiesbaden 2017; Barbara Flügge (a cura di), Smart Mobility in der Praxis: Das Auto - unverzichtbar für den intermodalen 
accrescere la qualità di vita, la sicurezza, le misure ecologico-ambientali, il risparmio energetico, raggiungendo il maggior numero possibile di persone di una città o di una determinata zona.

Un aspetto fondamentale della smart mobility è peraltro l'inclusione sociale. Per raggiungere tale obiettivo le persone che circolano nella città - siano essi cittadini, pendolari o semplicemente turisti e visitatori - devono essere messe nella condizione di avere un agevole accesso ai differenti modi di trasporto sostenibili e a possibilità di spostamento alternative. Ciò è reso possibile solo se questi soggetti ottengono in tempo reale informazioni personalizzate e corrette, così da poter organizzare al meglio i propri trasferimenti. ${ }^{23}$ Mediante la diffusione di dispositivi mobili come smartphone, ${ }^{24}$ tablet, notebook, pc portatili ecc. connessi ad internet, le persone nella smart city diventano al tempo stesso sensori e fonte di informazione per la comunità, dando feedback dinamici sullo stato del traffico e dei trasporti, per esempio riportando che le corriere sono al completo, che i treni necessitano di pulizie, che vi è coda su una determinata rotta stradale, segnalando indicenti e altri eventi stradali. ${ }^{25}$ L'utente dei trasporti nella smart mobility non può essere, quindi, mero soggetto passivo, bensì deve diventare un attore che gestisce la propria mobilità, informandosi per esempio sui tempi reali di percorrenza, sulle condizioni del traffico, sui vari modi di trasporto a disposizione.

In estrema sintesi, la mobilità della smart city si sostanzia in tre azioni chiave:

a) interoperabilità (ICT), ${ }^{26}$

b) sistemi di trasporto intelligenti (STI o $\operatorname{ITS}^{27}$ ),

c) mobilità sostenibile.

Per interoperabilità si intende, nel contesto della smart mobility, l'applicazione di tecnologie in grado di raccogliere mediante sensori tutte le informazioni e i dati indispensabili per far funzionare la mobilità e il trasporto. Questi dati dovranno provenire da tutti i mezzi in circolazione, da sistemi di tracciamento dei veicoli, da infrastrutture digitali a sostegno alla circolazione di merci, da reti di videosorveglianza, ma anche - come si è accennato - da strumenti di connessione tra utenti che dovranno operare come una grande social network for mobility and transport. In un secondo momento tutta questa ingente massa di dati dovrà confluire, in flusso cont-

Verkehr?, Wiesbaden 2018; Mahmoud Hashem Eiza/Yue Cao/Lexi Xu (a cura di), Toward Sustainable and Economic Smart Mobility: Shaping The Future Of Smart Cities, London 2020.

${ }^{23}$ Per una esposizione sintetica della neccessità dello scambio di informazioni e dati nella smart mobility v. Ingo Schwarzer, Smart Data for Mobility - Wie Daten unsere Mobilitaet veraendern, in: Internationales Verkehrswesen, 1, 2017-2, pp. 72 ss.

${ }^{24} \mathrm{Cfr}$. Anne Aguilera/Virginie Boutueil, Urban Mobility and the Smartphone: Transportation, Travel Behavior and Public Policy, Amsterdam 2019.

${ }^{25}$ Cfr. OICE, Smart City: Uno strumento per le Comunità Intelligenti, marzo 2017, Pomerzia - Roma, p. 91; online: file://server-01/User\$/ChristophP/Downloads/OICE_smart_web\%20(3).pdf e anche https://www.oice.it/516146/smart-city-uno-strumento-per-le-comunit-intelligenti (30.05.2020).

${ }^{26} \mathrm{ICT}=$ Information and Communications Technology.

${ }^{27}$ IST $=$ Intelligent Transport Systems o anche Intelligent Transportation Systems. 
inuo giorno e notte, in centrali operative in grado di gestire, elaborare e valorizzare questi dati in modo da fornire in un terzo momento a tutti gli utenti, siano essi passeggeri privati o trasportatori di merci, informazioni utili per la mobilità aggiornate in tempo reale. In tal modo dovrebbero migliorare la viabilità, la sicurezza, la qualità della vita, l'efficienza dell'impiego di risorse energetiche e la salvaguardia dell ambiente (per esempio, qualità dell aria). Per il funzionamento di questo tipo di mobilità, saranno pertanto fondamentali open data, cloud computing, smart devices (ovvero apparecchiature intelligenti di tutti i tipi) come anche e forse fondamentalmente l'internet of things [and services]. ${ }^{28}$

Per sistemi di trasporto intelligenti si intendono tutte le tecnologie - e sono in continuo aumento - finalizzate ad acquistare, processare, elaborare e diffondere le informazioni sul traffico a tutti i soggetti che ne vogliono fare uso, garantendo la interoperabilità sopra descritta. Si tratta di sistemi in grado di gestire il traffico e la mobilità, sistemi per l'informazione agli utenti, sistemi per la gestione del trasporto collettivo, sistemi per l'integrazione modale e sistemi per la gestione delle emergenze e degli incidenti.

Con mobilità sostenibile, infine, si fa riferimento all'insieme di tutti i sistemi di trasporto a basso impatto ambientale, economico e sociale, ma che comunque riescono a garantire una efficienza e rapidità possibilmente maggiore dei vecchi sistemi. Passi importanti in questo senso sono il potenziamento dell'utilizzo del trasporto pubblico locale e l'incremento di veicoli capaci di ridurre emissioni inquinanti ed emissioni acustiche, ma anche la creazione di strutture che diano sicurezza ai pedoni e lo sviluppo del sistema di mobilità urbano con delle piste ciclabili. ${ }^{29}$

\section{La struttura della smart mobility}

In sostanza vi sono quattro ambiti nei quali si sviluppa la struttura portante delle smart cities in materia di trasporto e mobilità; questi sono i seguenti:

a) la creazione di un'infrastruttura, costituita da reti e dotazioni tecnologiche, idonea a garantire un'efficiente

i. mobilità pubblica (per esempio nuove tecnologie per il trasporto pubblico locale - TPL),

ii. mobilità privata (per esempio controllo degli accessi alle zone a traffico limitato - ZTL, sosta a raso) e

iii. mobilità alternativa (per esempio stazioni/colonnine di ricarica per veicoli elettrici (e-mobility), ${ }^{30}$ modelli che si ispirano alla sharing economy come il

\footnotetext{
${ }^{28}$ Cfr. OICE, Smart City: Uno strumento per le Comunità Intelligenti, marzo 2017, Pomerzia Roma, p. 91-92.

${ }^{29}$ Cfr. ibid., p. 92-93.

${ }^{30} \mathrm{Cfr}$. Oliviero Baccelli/Raffaele Galdi/Gabriele Grea, L'e-mobility: Mercati e policies per un'evoluzione silenziosa, Milano 2016.
} 
bike/carsharing o car-pooling e la realizzazione delle relative infrastrutture come le piste ciclabili);

b) la creazione di un sistema sensoristico in grado di raccogliere i big data di tutti gli oggetti e soggetti connessi nella smart city, ovvero dati sull infrastruttura, sui veicoli circolanti, su ambiente, situazioni metereologiche e comportamenti degli utenti (per esempio reti IoT, reti stradali intelligenti con rilevatori del traffico, semafori intelligenti, autodetector, monitoraggio del TPL);

c) la creazione di una piattaforma di fornitura di servizi ovvero di una piattaforma che sia in grado elaborare, valorizzare e sviluppare i big data raccolti per migliorare la fornitura di servizi (per esempio centrali urbane per la mobilità, open data, geoportali a supporto della mobilità);

d) la creazione di applicazioni e la gestione dei servizi, infatti, deve essere creato un punto di contatto con l'utenza affinché questa, tramite web o mobile, possa accedere alle informazioni necessarie per poter usufruire dei servizi legati alla mobilità a al trasporto (per esempio bigliettazione elettronica, informazioni all'utenza, pagamenti elettronici per TPL, ZTL, parcheggi).

Questi ambiti vanno comunque implementati all'interno di un programma strategico, che ponga le esigenze della cittadinanza ovvero dello smart citizen al centro di ogni pianificazione. Se si vogliono raggiungere gli obbiettivi di riduzione del traffico, di potenziamento delle reti di mobilità sostenibile e di incremento della qualità degli spazi pubblici, a beneficio appunto dello smart citizen, è pertanto chiaro che tutte le iniziative edilizie ed urbanistiche di una città debbano essere pianificate tenendo conto anche della mobilità e dell'accessibilità.

Negli ultimi anni abbiamo percepito il fenomeno smart mobility attraverso la pianificazione adottata da molte città italiane, le quali si sono munite del Piano Urbano della Mobilità Sostenibile (PUMS). Le discussioni sorte fra politica e cittadinanza nel contesto del PUMS - come vedremo - pone l'attenzione su molti fenomeni che caratterizzano le basi di una smart mobility moderna e al contempo sono espressione di una nuova visione delle nostre città. 


\section{La smart mobility è già approdata nella pianificazione delle nostre città}

\subsection{Piani Urbani del Traffico}

In Italia solo nei Comuni con una popolazione di più di $30.000^{31}$ abitanti è obbligatoria $^{32}$ l' adozione di un Piano Urbano del Traffico (PUT), finalizzato al miglioramento delle condizioni di circolazione e della sicurezza stradale, alla riduzione dell'inquinamento acustico ed atmosferico ed al risparmio energetico. Il PUT previsto dall'art. 36 del Codice della Strada, ${ }^{33}$ il quale nell'ultima parte del comma quarto stabilisce, tra l'altro, che il Piano Urbano del Traffico preveda il ricorso ad adeguati sistemi tecnologici, su base informatica di regolamentazione e controllo del traffico, nonché di verifica del rallentamento della velocità e di dissuasione della sosta, al fine di consentire modifiche ai flussi della circolazione stradale in relazione agli obiettivi da perseguire.

Il Piano Urbano del Traffico deve essere redatto secondo le indicazioni contenute nel piano regolatore. Quest'ultimo, infatti, costituisce uno strumento urbanistico che si pone ad un livello superiore rispetto al piano urbano del traffico. ${ }^{34}$ Debbono, inoltre, essere qualificati come vincolanti solo gli interventi a breve termine del piano urbano del traffico. Gli interventi a medio e lungo termine, invece, hanno natura meramente indicativa e programmatoria. ${ }^{35}$ Il Consiglio di Stato ha chiarito che $\mathrm{i}$ Comuni, nell'adozione del piano urbano del traffico, devono rispettare le direttive emanate dal Ministero dei lavori pubblici. ${ }^{36}$

\footnotetext{
${ }^{31}$ Tale numero della popolazione non è riferito solo alla popolazione residente. Sono compresi nell' obbligo anche Comuni con una popolazione residente inferiore, i quali solo in alcuni mesi dell' anno per particolare affluenza turistica raggiungano tale numero; cfr. art. 36, co 2 Decreto legislativo, 30 aprile 1992, n. 285: "Nuovo codice della strada", in: Gazzetta Ufficiale, 18.05.1992, n. 114.

${ }^{32}$ Oltre ai Comuni, anche le Province e le Città metropolitane hanno l'obbligo di adottare gli appositi piani del traffico per la viabilità extraurbana, ossia un insieme di interventi per l'organizzazione della circolazione sulle strade che si trovano fuori dei centri abitati, cfr. art. 36, co. 1 Decreto legislativo. n. 285/1992.

${ }^{33}$ Decreto legislativo n. 285/1992.

${ }^{34}$ Cfr. T.A.R. Campania, Napoli, sez. V, sentenza 7 gennaio 2002, n. 185.

${ }^{35}$ Cfr. T.A.R. Trentino Alto Adige, Trento, sentenza 4 marzo 2002, n. 87.

${ }^{36}$ Cfr. Cons. Stato, sez. VI, sentenza 8 marzo 2006, n. 1270.
} 


\subsection{Piani Urbani di Mobilità}

A differenza del PUT, il Piano Urbano di Mobilità (PUM), introdotto nel nostro ordinamento dall ’art. 22 della Legge n. 340/2000, ${ }^{37}$ è uno strumento del quale l'amministrazione comunale può dotarsi volontariamente senza esserne obbligata. Il PUM va inteso come un progetto del sistema della mobilità di un Comune comprendente l'insieme organico degli interventi sulle infrastrutture di trasporto pubblico e stradali, sui parcheggi di interscambio, sulle tecnologie, sul parco veicoli e sul governo della domanda di trasporto.

Il PUM va poi attuato avvalendosi dei responsabili della mobilità (c.d. mobility manager), ${ }^{38}$ mediante sistemi di controllo e regolazione del traffico, con offerta di informazioni all'utenza, attraverso una adeguata logistica e tecnologie idonee alla riorganizzazione della distribuzione delle merci nelle città.

La finalità del PUM è quella di soddisfare i fabbisogni di mobilità della popolazione, assicurare l'abbattimento dei livelli di inquinamento atmosferico e acustico, la riduzione dei consumi energetici, l'aumento dei livelli di sicurezza del trasporto e della circolazione stradale, la minimizzazione dell'uso individuale dell'automobile privata e la moderazione del traffico, l'incremento della capacità di trasporto, l'aumento della percentuale di cittadini trasportati dai sistemi collettivi anche con soluzioni di car pooling e car sharing e la riduzione dei fenomeni di congestione nelle aree urbane. ${ }^{39}$

\subsection{Piano Urbano della Mobilità Sostenibile}

Quando un Comune o una città intende munirsi di un PUM che ponga una particolare accentuazione sulla mobilità sostenibile, il piano viene definito Piano Urbano della Mobilità Sostenibile (PUMS).

\footnotetext{
${ }^{37}$ V. art. 22 Legge, 24 novembre 2000, n. 340: "Disposizioni per la delegificazione di norme e per la semplificazione di procedimenti amministrativi - Legge di semplificazione 1999", in: Gazzetta Ufficiale, 24 novembre 2000, n. 275.

${ }^{38}$ La figura del responsabile della mobilità per enti pubblici con più di 300 dipendenti per unità locale è stato introdotto dall'art. 3 del Decreto interministeriale del Ministro dell'ambiente, del Ministro dei lavori pubblici, del Ministro della sanità, del Ministro dei trasporti e della navigazione 27 marzo 1998: "Mobilità sostenibile nelle aree urbane", in: Gazzetta Ufficiale, 3 agosto 1998, n. 179.

${ }^{39}$ Cfr. art. 22, co. 1, Legge n. 340/2000.
} 


\subsubsection{PUMS a livello dell'Unione Europea}

Da alcuni anni la Commissione Europea - e più precisamente il Commissario ai Trasporti - si occupa dei PUMS (Sustainable Urban Mobility Plans - SUMPs) ${ }^{40}$ definendoli una pietra angolare della propria politica della mobilità urbana e raccomandando vivamente a tutte le città europee di qualsiasi dimensione di munirsi di un PUMS. La Commissione invita attivamente gli Stati membri a promuovere i PUMS a livello nazionale, fornendo agli enti territoriali un idoneo supporto ed emanando normative adeguate. ${ }^{41}$

A livello tecnico la promozione dei PUMS rientra pertanto tra le competenze della Direzione Generale per la Mobilità e i Trasporti (DG MOVE), la quale organizza a riguardo corsi di formazione, diffonde esempi di good practice, crea opportunità di networking e fornisce possibilità di cofinanziamento come anche una piattaforma di coordinamento per i progetti relativi ai PUMS.

Le linee guida Guidelines for developing and implementing a Sustainable Urban Mobility Plan approvate dalla Direzione Generale per la Mobilità e i Trasporti della Commissione Europea, giunti ormai alla seconda edizione nell'ottobre 2019, sono pubblicate da European Local Transport Information Service (ELTIS ${ }^{42}$ e definiscono i PUMS nel seguente modo:

"A Sustainable Urban Mobility Plan is a strategic plan designed to satisfy the mobility needs of people and businesses in cities and their surroundings for a better quality of life. It builds on existing planning practices and takes due consideration of integration, participation, and evaluation principles ${ }^{43}$ ".

\footnotetext{
${ }^{40} \mathrm{Cfr}$. Comunicazione della Commissione del 17 dicembre 2013 al Parlamento europeo, al Consiglio, al Comitato economico e sociale europeo e al Comitato delle regioni: Insieme verso una Mobilità Urbana Competitiva ed efficace sul piano delle risorse, COM(2013)913 def., online: https:// eur-lex.europa.eu/legal-content/IT/TXT/?uri=CELEX\%3A52013DC0913 (30.05.2020).

${ }^{41} \mathrm{Cfr}$. https://ec.europa.eu/transport/themes/clean-transport-urban-transport/urban-mobility/urbanmobility-actions/sustainable-urban_en?2nd-language=ro (30.05.2020).

${ }^{42}$ Cfr. https://www.eltis.org/mobility-plans/sump-guidelines (30.05.2020).

${ }^{43}$ Cfr. European Platform on Sustainable Urban Mobility Plans (a cura di), Guidelines for developing and implementing a Sustainable Urban Mobility Plan, 2 ed., Bruxelles 2019, p. 9, online: https://www.eltis.org/sites/default/files/sump-guidelines-2019_mediumres.pdf (30.05.2020). Traduzione: "Piano Urbano della Mobilità Sostenibile è un piano strategico che si propone di soddisfare la variegata domanda di mobilità delle persone e delle imprese nelle aree urbane e peri-urbane per migliorare la qualità della vita nelle città. Il PUMS integra gli altri strumenti di piano esistenti e segue principi di integrazione, partecipazione, monitoraggio e valutazione".
} 


\subsubsection{PUMS a livello nazionale}

In Italia il 4 agosto 2017 veniva emanato il Decreto del Ministero delle Infrastrutture e dei Trasporti ${ }^{44}$ n. 397, il quale aveva il proprio fondamento legislativo nell' art. 3, comma 7 lett. C) del decreto legislativo n. 257/2016, ${ }^{45}$ recante le linee guida per $i$ piani urbani di mobilità sostenibile. Ai sensi di tale decreto, le Città metropolitane, gli enti di area vasta, i Comuni e le associazioni di Comuni con popolazione superiore a 100.000 abitanti sono tenuti a predisporre e adottare i nuovi PUMS, secondo le linee guida ministeriali, entro ventiquattro mesi dall'entrata in vigore del decreto. Tale è obbligo è stato prorogato. ${ }^{46}$

La finalità di questo decreto è di favorire l'applicazione omogenea e coordinata delle linee guida per la redazione di Piani Urbani di Mobilità sostenibili su tutto il territorio nazionale. Un PUMS, secondo il decreto, deve avere come obiettivi principali il miglioramento dell'accessibilità alle aree urbane e periurbane, mediante sistemi di mobilità e trasporti sostenibili e di alta qualità anche sotto il profilo ambientale, economico e sociale, ed il miglioramento della fruibilità dello spazio pubblico. ${ }^{47}$ Esso deve pertanto promuovere la mobilità sostenibile, il che significa che deve orientare la mobilità dei residenti e dei city user in modo che questi possano privilegiare gli spostamenti a piedi, in bicicletta o con mezzi pubblici ovvero utilizzare mezzi privati a basso impatto ambientale, nonché creare le infrastrutture che consentano il miglior utilizzo delle stesse verso, attraverso e all'interno delle aree urbane e periurbane. ${ }^{48}$ Nell'individuazione delle strategie e delle azioni necessarie per raggiungere gli obbiettivi posti dal PUMS, nel DM n. 397/2017 vengono considerati molti aspetti importanti per la realizzazione di una smart mobility nelle città italiane, come per esempio:

- l'introduzione di sistemi di mobilità motorizzata condivisa, quali car-sharing, bike-sharing, van-sharing, car-pooling e sviluppo di politiche tariffarie in favore di car sharing, moto sharing e carpooling e agevolazione di transito e sosta per i veicoli con mobilità condivisa; l'implementazione di servizi di bike sharing (bicycle commuting) anche per turisti e utenti occasionali creando infrastrutture adeguate come le piste ciclabili;

\footnotetext{
${ }^{44}$ Cfr. Decreto del Ministero delle Infrastrutture e dei Trasporti 4 agosto 2017, n. 397: "Individuazione delle linee guida per i piani urbani di mobilità sostenibile, ai sensi dell'articolo 3, comma 7, del decreto legislativo 16 dicembre 2016, n. 257.”, in: Gazzetta Ufficiale, 5 agosto 2017, n. 233.

${ }^{45} \mathrm{Cfr}$. Decreto Legislativo, 16 dicembre 2016, n. 257: "Disciplina di attuazione della direttiva 2014/94/UE del Parlamento europeo e del Consiglio, del 22 ottobre 2014, sulla realizzazione di una infrastruttura per i combustibili alternativi.”, in: Gazzetta Ufficiale, 13 gennaio 2017, n. 10.

${ }^{46} \mathrm{Cfr}$. Decreto del Ministero delle Infrastrutture e dei Trasporti 28 agosto 2019, n. 396: "Modifiche delle linee guida per la redazione dei PUMS di cui al Decreto del Ministero delle Infrastrutture e dei Trasporti 4 agosto 2017, n. 397", in: Gazzetta Ufficiale, 30 ottobre 2019, n. 255, il quale proroga il termine ultimo originariamente al 20 ottobre 2020.

${ }^{47} \mathrm{Cfr}$. allegato 2, comma 1, Decreto del Ministero delle Infrastrutture e dei Trasporti 28 agosto 2019, n. 396.

${ }^{48} \mathrm{Cfr}$. ibid.
} 
- l'utilizzo di sistemi di trasporto intelligenti (ITS) da parte degli operatori del trasporto pubblico, attraverso l'incremento nella dotazione di veicoli di sistemi per il monitoraggio in tempo reale della localizzazione e del servizio finalizzato ad adeguare gli orari del servizio alla domanda effettiva di passeggeri e ad intervenire anche in tempo reale per modifiche dei piani di esercizio (centrale operativa, AVM-Automatic Vehicle Monitoring, e AVL-Automatic Vehicle Location);

- la rilevazione del numero di passeggeri a bordo, attraverso l'installazione di dispositivi sui mezzi, con l'avvio di sperimentazioni specifiche per l'utilizzo della telefonia mobile;

- l'utilizzo diffuso dei diversi canali di comunicazione all'utenza: informazioni a bordo e alle fermate; siti web informativi; social network come Facebook e Twitter; telefoni cellulari, mediante SMS di avviso;

- applicazioni per smartphone; schermi e altoparlanti nelle stazioni e presso le fermate e all'interno delle vetture; schermi e computer touch-screen in luoghi strategici come ospedali, centri commerciali e università; pannelli a messaggio variabile;

- la creazione di corsie preferenziali per il TPL su gomma e l'implementazione di impianti semaforici asserviti e preferenziali al TPL;

- lo sviluppo dell'integrazione tariffaria prevedendo anche il trasporto delle biciclette sui mezzi del TPL, sui treni e sui traghetti;

- l'utilizzo dell'ITS e di sistemi di infomobilità per favorire l'integrazione di sistemi di trasporto, per la fornitura di dati sulla rete prioritaria urbana e per lo sviluppo di servizi innovativi di mobilità;

- l'utilizzo di ITS e piattaforme software in grado di gestire il trasporto privato condiviso e di integrarlo con il TPL;

- la diffusione di sistemi ettometrici automatizzati, segnaletica way finding e dispositivi d'ausilio alla mobilità dell'utenza debole (semafori con segnalazione acustica, scivoli, percorsi tattili, ecc.);

- l'installazione di colonnine per la ricarica elettrica e impianti per la distribuzione di combustibili alternativi a basso impatto inquinante.

\section{L' Ordinamento dell'Unione Europea}

La politica comune dei trasporti rientra nella competenza concorrente dell'Unione europea ${ }^{49}$ ovvero in uno dei tanti settori - e sono la maggior parte all'interno dell'ordinamento dell Unione - nei quali la competenza a legiferare e ad adottare atti giuridicamente vincolanti spetta tanto all'Unione quanto agli Stati membri. ${ }^{50} \mathrm{~A}$ ben

\footnotetext{
${ }^{49} \mathrm{~V}$. art. 4, par. 2 lit. g) e h) TFUE.

${ }^{50}$ Per una panoramica della politica comune dei trasporti dell'UE v. Carola Pagliarin, La governance dei trasporti. Nuove prospettive tra integrazione europea e regionalismo differenziato, in: Carola Pagliarin/Christoph Perathoner/Simon Laimer (a cura di), Per una Europa più unita nel settore dei trasporti - Assetti istituzionali, economici e normativi. Il diritto dei trasporti nell'Uni-
} 
vedere però il mero aspetto trasportistico nella smart mobility non è l'essenziale, ma va visto come elemento funzionale per la realizzazione della smart city. La smart mobility comprende dunque da un lato un insieme di innovazioni trasportistiche volte ad aumentare la qualità di vita, la sicurezza, le misure ecologico-ambientali, il risparmio energetico ecc., ma dall altro lato rappresenta anche - come abbiamo visto sopra - una visione divenuta strumento indispensabile per la pianificazione urbana. L'Unione Europa non ha una competenza diretta nelle politiche urbane, ciononostante, trattando la smart city nella sua multidimensionalità, ed in particolare soffermandosi su ciò che è la smart mobility, si nota come quest'ultima costituisca di fatto da anni un punto nevralgico di un'azione europea che mira ad una crescita e ad uno sviluppo della mobilità sostenibili e sensibili rispetto alle problematiche globali, soprattutto ambientali, che anche l'Unione è chiamata ad affrontare.

A livello internazionale sono ravvisabili dal punto di vista giuridico due modelli di riferimento per la realizzazione delle smart cities. Il primo, che può definirsi bottom-up e che può valere come modello classico degli Stati Uniti, parte dal presupposto che l'innovazione consiste in un processo dal basso verso l'alto e che le autorità pubbliche non si debbano inserire in questo processo. L'autorità pubblica regolamenta pertanto solo lo stato di fatto che si è prodotto al termine del processo di innovazione, essendo così al massimo chiamata a promuovere un quadro normativo che favorisce le nuove tecnologie che si affacciamo sul mercato spesso, ma non sempre, nella veste di start-up. Il secondo modello, che è quello praticato nell'Unione Europea, si differenzia sostanzialmente dal sistema statunitense, perché è proprio l'opposto. Esso presuppone che sia l'autorità pubblica, dall'alto, a determinare certi traguardi ed individuare delle regolamentazioni tali da favorire tecnologie particolari ed incentivare innovazioni. In questo secondo modello le pubbliche amministrazioni, a tutti i livelli, vengono a loro volta incentivate ad impiegare determinati prodotti e determinate tecnologie nel settore dei trasporti. Si tratta pertanto di un modello top-down. ${ }^{51}$

Del resto, il tema della sostenibilità e dello sviluppo sostenibile, che si pone alla base dell'approccio smart, anima ormai da decenni i dibattiti in sede di conferenze internazionali, ${ }^{52}$ spingendo i Paesi, sin dagli anni Settanta del secolo scorso, a riconoscerne la crescente importanza e ad abbracciare le soluzioni che essa è in grado di offrire.

one europea, Milano, 2020, pp. 201 ss.; Christoph Perathoner, Il diritto dei trasporti nel mercato interno europeo e nella sua dimensione esterna, in: ibid., pp. 1 ss.

${ }^{51}$ Cfr. Edoardo Ferrero, Le smart cities nell' ordinamento giuridico, in: Foro Amministrativo, 2015, 4, pp. $1272 \mathrm{~s}$.

${ }^{52} \mathrm{Cfr}$. Carmela Gargiulo, Integrazione trasporti-territorio. Strumenti, interventi, e best practices verso la Smart City, Napoli, 2014, pp. 15 s.; vedi in particolare anche Conferenza delle Nazioni Unite sull'ambiente umano, tenutasi a Stoccolma, tenutasi tra il 5 e il 16 giugno 1972, consultabile online: http://www.un-documents.net/aconf48-14r1.pdf (30.05.2020) e per il concetto di "sostenibilità" v. Donella H. Meadows/Dennis L. Meadows/Jgrgen Randers/William W. Behrens III (a cura di), The Limits to Growth: A report for the Club of Rome's Project on the Predicament of Mankind, A Potomac Associates Book, New York, 1972. 
Così, per ciò che attiene alla mobilità sostenibile, prendendo le mosse da quanto previsto dall'A genda $21,{ }^{53}$ che aveva individuato nel trasporto, oltre che il motore dello sviluppo sociale ed economico, una delle più gravi minacce ambientali, la Commissione europea aveva elaborato il Libro Bianco del 1992 sui trasporti "Lo sviluppo futuro della politica dei trasporti. Una strategia globale per la realizzazione di un quadro comunitario atto a garantire una mobilità sostenibile", ${ }^{54}$ gettando di fatto le fondamenta per una politica trasportistica sostenibile. In tale scritto si legge come l'obiettivo da perseguire per garantire uno sviluppo sostenibile sia la realizzazione di un sistema di trasporto efficiente e sicuro, che si dispieghi su reti transeuropee, sfruttando le migliori risorse tecnologiche disponibili, e a cui possano avere accesso, a costi ridotti, cittadini e imprese, promuovendo la coesione sociale. Con il Trattato di Maastricht sull'Unione europea (TUE), firmato il 7 febbraio $1992,{ }^{55}$ si accentuavano aspetti della mobilità quali la sicurezza e la compatibilità con l'ambiente e venivano introdotti i concetti di reti transeuropee (TEN) ${ }^{56}$ nei settori dei trasporti, dell'energia e delle telecomunicazioni, volte a collegare tutte le regioni e le città d'Europa.

Con la successiva comunicazione della Commissione nel luglio del 1995, inerente al programma d'azione da seguire per attuare la politica comune dei trasporti negli anni 1995-2000, ${ }^{57}$ veniva poi ribadita la volontà di realizzazione di una mobilità sostenibile, attraverso la creazione di un sistema trasportistico efficiente, accessibile e competitivo, nonché rispettoso dell'ambiente e sicuro. Le azioni principali venivano individuate sia a livello del mercato interno, tramite lo sviluppo di sistemi di trasporto integrati e competitivi basati su tecnologie avanzate, sia nella dimensione esterna verso i Paesi terzi.

Grazie alla pubblicazione di un nuovo e per molti aspetti fondamentale Libro Bianco del 2001 sui trasporti, ${ }^{58}$ la Commissione europea incentivava un'integrazione

\footnotetext{
${ }^{53}$ Documento di intenti e obiettivi programmatici su ambiente, economia e società sottoscritto da oltre 170 Paesi di tutto il mondo durante la conferenza delle Nazioni Unite su Ambiente e Sviluppo (United Nations Conference on Environment and Development; UNCED) tenutasi a Rio de Janeiro tra il 3 e il 14 giugno 1992; v. https://www.minambiente.it/pagina/lagenda-21 (30.05.2020).

${ }^{54}$ Cfr. Comunicazione della Commissione delle Comunità europee del 2 dicembre 1992: "Lo sviluppo futuro della politica dei trasporti. Una strategia globale per la realizzazione di un quadro comunitario atto a garantire una mobilità sostenibile", $\operatorname{COM}(92) 494$ def., in: Bollettino delle Comunità europee, supplemento 3/93, consultabile online anche in lingua italiana: https://op.europa.eu/ en/publication-detail/-/publication/67d2cd43-9740-42b0-8ba8-e759d36f3109 (30.05.2020) o anche https://eur-lex.europa.eu/legal-content/IT/TXT/PDF/?uri=CELEX:51992DC0494\&from=IT (30.05.2020).

${ }^{55}$ Entrato in vigore il 7 febbraio 1992; v. Trattato sull'Unione europea, in: GUCE, 29 luglio 1992, C 191, pp. 1 ss., ELI: http://data.europa.eu/eli/treaty/teu/sign (30.05.2020).

${ }^{56} \mathrm{TEN}=$ Transeuropean Network (TEN-T = Trans-European Transport Network); v. artt. 170-172 TFUE.

${ }^{57} \mathrm{Cfr}$. Comunicazione della Commissione del 12 luglio 1995 al Consiglio, al Parlamento europeo, al Comitato economico e sociale e al Comitato delle regioni: "La politica comune dei trasporti: programma d'azione 1995-2000", COM(95)302def., online: https://eur-lex.europa.eu/legal-content/IT/TXT/PDF/?uri=CELEX:51995DC0302\&from=EN (30.05.2020).

${ }^{58} \mathrm{Cfr}$. Libro Bianco della Commissione europea del 12 settembre 2001: "La politica europea dei
} 
tra le varie modalità trasportistiche, promuovendo tra l'altro l'intermodalità e lanciando diversi progetti in tal senso, senza però elaborare atti normativi volti alla realizzazione degli obiettivi prefissati.

Diverse sono le comunicazioni della Commissione che hanno fatto seguito al Libro Bianco del 2001, tra cui è utile citare, in relazione allo sviluppo dell'ideologia di una smart mobility, quella relativa ad una Strategia tematica sull'ambiente urbano $^{59}$ e quella riguardante la politica di coesione e le città. ${ }^{60}$ Nella prima si mette anzitutto in luce la centralità delle aree urbane, motori dell'economia europea, rilevando però come la qualità della vita dei cittadini europei sia sempre più compromessa e messa a rischio dalle crescenti problematiche ambientali quali l'inquinamento dell'aria e acustico, il traffico e le congestioni, il cattivo sfruttamento dello spazio edificato e la produzione di rifiuti. L'Unione si prefiggeva così di promuovere buone pratiche, incentivando uno scambio di esperienze tra le città e sottolineando la necessità di un'azione su misura a livello locale, coerentemente con il principio di sussidiarietà; la seconda comunicazione inserisce tra i punti strategici finalizzati al rafforzamento dell'attrattività delle città i trasporti, l'accessibilità e la mobilità, proponendo, tra l'altro, il coordinamento tra la varie modalità trasportistiche, il collegamento alle reti transeuropee di trasporto, una strategia integrata dei trasporti per la zona urbana e l'adozione di un approccio integrato che favorisca alternative valide ai veicoli maggiormente inquinanti.

Più tardi, veniva ribadita, tramite la pubblicazione del Libro Verde del 2007 sul trasporto ${ }^{61}$ la sensibilità europea verso la mobilità urbana, lanciando cinque sfide da vincere nell' ambito di un approccio integrato, vale a dire la realizzazione di un traffico più scorrevole nelle città, di una città più pulita, di un trasporto urbano più intelligente, di un trasporto urbano accessibile e di un trasporto urbano sicuro. Relativamente alla sfida per un trasporto urbano più intelligente, la Commissione individuava nello sfruttamento degli ITS una valida opzione di gestione ottimale e dinamica dei servizi; concretamente, tramite la gestione del parco veicoli, i sistemi di informazione agli utenti, le biglietterie automatiche e le smart cards per i pagamenti, intercambiabili per i diversi modi di trasporto, l'utente delle reti di mobilità urbane sarebbe incentivato a servirsene maggiormente, essendo in grado di compiere una scelta informata.

trasporti fino al 2010: il momento delle scelte", $\operatorname{COM}(2001) 370$ def., online: http://www.mit.gov. it/mit/mop_all.php?p_id=04863 (30.05.2020).

${ }^{59} \mathrm{Cfr}$. Comunicazione della Commissione del 11 gennaio 2006 al Consiglio e al Parlamento Europeo relativa ad una strategia tematica sull'ambiente urbano, COM(2005)718 def., online: https:// eur-lex.europa.eu/legal-content/IT/TXT/?uri=CELEX:52005DC0718 (30.05.2020).

${ }^{60} \mathrm{Cfr}$. Comunicazione della Commissione del 13 luglio 2006 al Consiglio e al Parlamento europeo: "La politica di coesione e le città: il contributo delle città e degli agglomerati urbani alla crescita e all'occupazione all'interno delle regioni", $\operatorname{COM}(2006) 385$ def., online: https://eur-lex.europa.eu/ legal-content/IT/TXT/?uri=CELEX:52005DC0299 (30.05.2020).

${ }^{61}$ Libro Verde della Commissione europea del 25 settembre 2007: "Verso una nuova cultura della mobilità urbana", COM(2007)551 def., online: https://eur-lex.europa.eu/legal-content/IT/LSU/?uri=CELEX\%3A52007DC0551 (30.05.2020). 
Un'attenzione particolare alla tematica della sostenibilità, della modernizzazione delle reti infrastrutturali urbane e del miglioramento dell'efficienza energetica si pose, peraltro, sempre nel 2007, da parte degli Stati membri su iniziativa della Presidenza tedesca con la pubblicazione della Carta di Lipsia sulle Città Europee Sostenibili, ${ }^{62}$ recante strategie e principi comuni per una politica di sviluppo urbano integrata ed inclusiva e alla base di una serie di programmi finanziati dall'Unione, quali URBACT ${ }^{63}$ e Urban Innovative Actions. ${ }^{64}$

Un ulteriore passo avanti compiuto in sede europea è rappresentato dal Piano d'azione sulla mobilità urbana ${ }^{65}$ che evidenzia la necessità di una collaborazione a livello comunitario tra le autorità locali, regionali e nazionali, in un'ottica di coerenza e di incoraggiamento e sostegno alle politiche di mobilità urbana sostenibili, tramite scambio di buone pratiche ed erogazione di finanziamenti.

Pare di estrema importanza, inoltre, la Comunicazione della Commissione del $2010,{ }^{66}$ inerente al piano d'azione relativo alle applicazioni del sistema globale di radionavigazione via satellite $(G N S S),{ }^{67}$ comprendente i sistemi di segnalazione per uso civile $G A L I L E O^{68}$ ed $E G N O S,{ }^{69}$ la cui applicazione nel settore dei trasporti, per

${ }^{62}$ Per una traduzione italiana della bozza finale della Carta di Lipsia adottata in occasione della riunione informale dei ministri per lo Sviluppo urbano e la coesione territoriale tenutasi a Lipsia/ Leipzig il 24 e 25 maggio 2007, CdR 163/2007 EN-COM/SAB/lc v. http://www.pdc.minambiente. it/sites/default/files/allegati/CdR_163_2007\%20EN_COM_SAB_lc.pdf (30.05.2020); per una scheda di lettura della Camera dei Deputati della Carta di Lipsia v. http://documenti.camera.it/ Leg15/Dossier/Testi/Po016.htm (30.05.2020).

${ }^{63} \mathrm{~V}$. https://urbact.eu (30.05.2020).

${ }^{64} \mathrm{~V}$. https://www.uia-initiative.eu/en/about-us/what-urban-innovative-actions (30.05.2020).

${ }^{65} \mathrm{Cfr}$. Comunicazione della Commissione del 30 settembre 2009 al Parlamento Europeo, al Consiglio e al Comitato economico e sociale europeo e al Comitato delle regioni: "Piano d'azione sulla mobilità urbana", COM (2009)490 def., online: https://eur-lex.europa.eu/legal-content/IT/ TXT/?uri=CELEX\%3A52009DC0490 (30.05.2020).

${ }^{66}$ Comunicazione della Commissione del 14 giugno 2010 al Consiglio, al Parlamento Europeo, al Comitato Economico e Sociale e al Comitato delle Regioni: "Piano d'azione relativo alle applicazioni del sistema globale di radionavigazione via satellite (GNSS)", $\operatorname{COM}(2010) 308$ def., online: https://eur-lex.europa.eu/legal-content/IT/TXT/?uri=CELEX:52010DC0308 (30.05.2020).

${ }^{67}$ GNSS $=$ Global Navigation Satellite System .

${ }^{68} \mathrm{Il}$ programma Galileo è un sistema di posizionamento e navigazione satellitare civile creato ad iniziativa dell'UE e dell'Agenzia Spaziale Europea per rendersi indipendenti dal sistema GPS (= NAVSTAR Global Positioning System = Navigation Satellite Timing And Ranging Global Positioning System) controllato dagli Stati Uniti. Nell 2004 è stata costituita la European Global Navigation Satellite Systems Agency (= The European GNSS Agency; GAS) ovvero l'Agenzia del GNSS europeo come agenzia dell'Unione europea avente dal 2012 sede a Praga; v. https://www.gsa.europa.eu/ (30.05.2020).

${ }^{69} \mathrm{EGNOS}=$ European geostationary navigation overlay system (= sistema geostazionario europeo di navigazione di sovrapposizione). Il sistema fu creato dalla collaborazione tra l'Agenzia Spaziale Europea (European Space Agency), la Commissione europea e EUROCONTROL. Si tratta di una sistema formato da delle basi terrestri collegate con una rete di satelliti per migliorare la precisione e affidabilità dei dati del sistema NAVSTAR GPS, ormai diventato indispensabile anche per il settore dei trasporti, si pensi all'aeronavigazione o alla navigazione marittima per rotte difficili; $\mathrm{v}$. https://www.esa.int/Applications/Navigation/Galileo_and_EGNOS (30.05.2020); v. anche Comunicazione della Commissione del 26.10.2016 al Parlamento europeo, al Consiglio, al Comitato 
esempio, per introdurre nuovi sistemi di pedaggio stradale e per una localizzazione precisa e in tempo reale di trasporti pubblici e mezzi di soccorso, comporta certamente benefici in termini di efficienza.

Fondamentale risulta poi la pubblicazione del Libro Bianco del 2011 sul trasporto ${ }^{70}$ in esso sono racchiusi ambiziosi progetti volti a gettare le fondamenta dello spazio europeo dei trasporti del futuro. Dieci sono gli obiettivi fissati da conseguire entro il 2030 e il 2050, tra cui spiccano la realizzazione di una rete essenziale TEN-T multimodale e di una rete di qualità e capacità elevate, con relativi servizi di informazione, e la definizione entro il 2020 di un quadro per un sistema europeo di informazione, gestione e pagamento nel settore dei trasporti multimodali.

Venendo agli atti normativi comunitari, la pietra miliare in tema di smart mobility è certamente rappresentata dalla Direttiva 40/2010/UE, ${ }^{71}$ che, sfruttando il progresso nel campo delle tecnologie e delle telecomunicazioni, si propone di diffondere ed incentivare le applicazioni dei sistemi di trasporto intelligenti - ITS tramite la predisposizione di servizi di informazioni sulla mobilità multimodale e sul traffico, attraverso la trasmissione di dati e le comunicazioni agli utenti di informazioni sui servizi stradali, nonché attraverso la predisposizione di un sistema di chiamata di emergenza ( $e$ Call) e di servizi di informazione e prenotazione per aree di parcheggio sicure per gli automezzi pesanti. Nella direttiva vengono, peraltro, presi in considerazione aspetti delicati quali la tutela della vita privata e il trattamento dei dati personali; al fine di garantire la compatibilità, l'interoperabilità e la continuità per la diffusione e l'utilizzo operativo degli ITS, viene prevista l'adozione di specifiche vincolanti per gli Stati membri da parte della Commissione.

Dalla succitata direttiva è derivata una pluralità di regolamenti delegati, disciplinanti nel dettaglio il servizio elettronico di chiamata di emergenza, ${ }^{72}$ le aree di parcheggio sicure per i mezzi pesanti, ${ }^{73}$ i dati e le procedure per la comunicazione

economico e sociale europeo e al Comitato delle regioni: "Strategia spaziale per l'Europa", COM (2016) 705 def., online: https://eur-lex.europa.eu/legal-content/IT/TXT/?uri=CELEX:52016DC0705 (30.05.2020).

${ }^{70} \mathrm{Cfr}$. Libro Bianco della Commissione europea del 28 marzo 2011: "Tabella di marcia verso uno spazio unico europeo dei trasporti - Per una politica dei trasporti competitiva e sostenibile", COM (2011)144 def., online: https://eur-lex.europa.eu/legal-content/IT/TXT/?uri=celex:52011DC0144 (30.05.2020).

${ }^{71}$ Direttiva 2010/40/UE del Parlamento europeo e del Consiglio, del 7 luglio 2010, sul quadro generale per la diffusione dei sistemi di trasporto intelligenti nel settore del trasporto stradale e nelle interfacce con altri modi di trasporto, in: GUUE, 6 agosto 2010, L 270, pp. 1 ss., ELI: http://data. europa.eu/eli/dir/2010/40/oj (30.05.2020).

${ }^{72}$ Regolamento delegato (UE) n. 305/2013 della Commissione del 26 novembre 2012, che integra la direttiva 2010/40/UE del Parlamento europeo e del Consiglio per quanto riguarda la predisposizione armonizzata in tutto il territorio dell'Unione europea di un servizio elettronico di chiamata di emergenza (eCall) interoperabile, in: GUUE, 3 aprile 2013, L 91/1, pp. 1 ss., ELI: http://data. europa.eu/eli/reg_del/2013/305/oj (30.05.2020).

${ }^{73}$ Regolamento Delegato (UE) n. 885/2013 della Commissione, del 15 maggio 2013, che integra la direttiva 2010/40/UE del Parlamento europeo e del Consiglio sui sistemi di trasporto intelligenti, in merito alla predisposizione dei servizi d'informazione sulle aree di parcheggio sicure destinate agli automezzi pesanti e ai veicoli commerciali, in: GUUE, 18 settembre 2013, L 274, pp. 1 ss., ELI: http://data.europa.eu/eli/reg_del/2013/885/oj (30.05.2020). 
gratuita agli utenti, ${ }^{74}$ i servizi di informazione sul traffico ${ }^{75}$ e di informazione sulla modalità multimodale. ${ }^{76}$

Con il continuo evolversi della tecnologia, percependo il parallelo cambiamento del settore dei trasporti, la Commissione europea ha emanato nel 2016 una nuova comunicazione $^{77}$ ponendo al centro dell' attenzione proprio l'applicazione delle tecnologie digitali nel sistema trasportistico, nonché individuando nello scambio di dati una possibilità per un uso ottimale ed efficiente delle risorse. Per ogni settore strategico del piano di diffusione dei servizi C-ITS ${ }^{78}$ - la sicurezza delle comunicazioni, la protezione dei dati personali, le tecnologie di comunicazione e frequenze, la interoperabilità su tutti i livelli, la valutazione della conformità e cooperazione internazionale - sono state previste azioni specifiche che definiranno il futuro del sistema di trasporto UE. L'esortazione agli Stati membri e all'industria è dunque quella di sostenere l'approccio adottato dalla Commissione.

Recentemente, nel 2018 la Commissione ${ }^{79}$ è tornata a sottolineare la centralità della tecnologia nei trasporti, e in particolare l'opportunità scaturente dalla

\footnotetext{
${ }^{74}$ Regolamento delegato (UE) n. 886/2013 della Commissione, del 15 maggio 2013, che integra la direttiva 2010/40/UE del Parlamento europeo e del Consiglio per quanto riguarda i dati e le procedure per la comunicazione gratuita agli utenti, ove possibile, di informazioni minime universali sulla viabilità connesse alla sicurezza stradale, in: GUUE, 18 settembre 2013, L 247, pp. 6 ss., ELI: http://data.europa.eu/eli/reg_del/2013/886/oj (30.05.2020).

${ }^{75}$ Regolamento delegato (UE) 2015/962 della Commissione, del 18 dicembre 2014, che integra la direttiva 2010/40/UE del Parlamento europeo e del Consiglio relativamente alla predisposizione in tutto il territorio dell'Unione europea di servizi di informazione sul traffico in tempo reale, in: GUUE, 23 giugno 2015, L 157, pp. 21 ss., ELI: http://data.europa.eu/eli/reg_del/2015/962/oj (30.05.2020).

${ }^{76}$ Regolamento delegato (UE) 2017/1926 della Commissione, del 31 maggio 2017, che integra la direttiva 2010/40/UE del Parlamento europeo e del Consiglio per quanto riguarda la predisposizione in tutto il territorio dell'Unione europea di servizi di informazione sulla mobilità multimodale, in: GUUE, 21 ottobre 2017, L 272, pp. 1 ss., ELI: http://data.europa.eu/eli/reg_del/2017/1926/oj (30.05.2020).

${ }^{77} \mathrm{Cfr}$. Comunicazione della Commissione del 30 novembre 2016 al Parlamento Europeo, al Consiglio, al Comitato Economico e Sociale europeo e al Comitato delle Regioni: "Una strategia europea per i sistemi di trasporto intelligenti cooperativi, prima tappa verso una mobilità cooperativa, connessa e automatizzata", COM (2016)766 def., online: https://eur-lex.europa.eu/legal-content/ IT/ALL/?uri=CELEX\%3A52016DC0766 (30.05.2020).

${ }^{78} \mathrm{C}$-ITS $=$ Cooperative Intelligent Transport Systems. C-ITS consentono ai veicoli stradali di interagire gli uni con gli altri e anche con l'infrastruttura stradale sulla quale stanno circolando. Nel trasporto su strada in particolare, i C-ITS comprendono (i) la comunicazione veicolo-veicolo (V2V = vehicle-to-vehicle $)$, (ii) la comunicazione veicolo-infrastruttura $(\mathrm{V} 2 \mathrm{I}=$ vehicle-to-infrastructure $)$ e/o (iii) la comunicazione infrastruttura-infrastruttura (I2I = infrastructure-to-infrastructure) come anche (iv) la comunicazione tra i veicoli e i pedoni o i ciclisti (V2X = vehicle-to-everything [veicolo con tutto]). La piattaforma C-Roads è un'iniziativa comune degli Stati membri dell'UE e dei gestori della rete stradale, finanziata da fondi ingenti messi a disposizione a livello nazionale e dell'UE, per poter testare in concreto ed attuare su strada servizi C-ITS, operando ai fini di una armonizzazione e dell'interoperabilità transfrontaliera; v. https://www.c-roads.eu/platform.html (30.05.2020).

${ }^{79}$ Comunicazione della Commissione del 17 maggio 2018: "Verso la mobilità automatizzata: una
} 
circolazione di veicoli cosiddetti autonomi (self driving cars). ${ }^{80}$ Gli obiettivi che si intendono raggiungere attraverso l'impiego diffuso della mobilità autonoma sono anzitutto la sicurezza, ${ }^{81}$ ma anche la riduzione di congestioni e la riduzione delle emissioni nocive, ${ }^{82}$ che per essere realizzati necessitano di un rafforzamento nel campo delle tecnologie e della cyber-sicurezza.

\section{Aspetti concreti della smart mobility in Europa e riflessioni conclusive}

La filosofia intrinseca al concetto delle smart cities offre certamente spazi idonei a sviluppare soluzioni innovative e al contempo efficaci di mobilità intelligente, favorendo un ecosistema collaborativo in grado di raggiungere obiettivi di sostenibilità.

La sfida che ogni città moderna è tenuta ad affrontare è chiara: la popolazione di ogni metropoli europea esige oggigiorno mezzi di trasporto sicuri, puliti, affidabili e convenienti per giungere da un posto all'altro e tornare. Al contempo, si chiede la riduzione del traffico stradale, degli incidenti e dell'inquinamento. Le strategie smart volte a fronteggiare le predette sfide e problematiche, rispettivamente lanciate

strategia dell'UE per la mobilità del futuro", COM (2018)283 def., online: https://eur-lex.europa. eu/legal-content/it/TXT/?uri=CELEX\%3A52018DC0283 (30.05.2020).

${ }^{80} \mathrm{Dal}$ punto di vista giuridico la smart mobility - quando si entra nel dettaglio - è accompagnata da una moltitudine di nuovi temi giuridici. Un ottimo esempio ne è la circolazione di veicoli autonomi ovvero la guida di automobili senza conducente. È evidente che in assenza di una conducente, qualora dovesse occorrere un incidente, si pongono nuove questioni di responsabilità. V. sulla questione l'UE https://ec.europa.eu/transparency/regexpert/index.cfm?do=groupDetail. groupMeetingDoc\&docid=36608 (30.05.2020) e l'OECD https://www.oecd-ilibrary.org/transport/automated-and-autonomous-driving_5jlwvzdfk640-en (30.05.2020); v. anche Bernhard A. Koch, Product Liability 2.0 - Mere Update or New Vision?, in: Sebastian Lohsse/Reiner Schulze/Dirk Staudenmayr (a cura di), Liability for Artificial Intelligence and the Internet of Things, Baden-Baden, pp. 99 ss.; Diana Cerini/Andrea Pisani Tedesco, Smart mobility, smart cars e intelligenza artificiale: responsabilità e prospettive (Collana del Dipartimento Giurisprudenza dell'Università di Milano-Bicocca), Torino, 2019; Francesco Di Ciommo, Evoluzione tecnologica e regole di responsabilità civile, Napoli, 2003.

${ }^{81}$ Cfr. Relazione della Commissione del 12 dicembre 2016 al Parlamento europeo e al Consiglio: "Salvare vite umane: migliorare la sicurezza dei veicoli nell'UE", Relazione sul monitoraggio e la valutazione delle caratteristiche avanzate di sicurezza dei veicoli, del loro rapporto costi-benefici e della fattibilità di una revisione dei regolamenti sulla sicurezza generale dei veicoli e sulla protezione dei pedoni e di altri utenti della strada vulnerabili, COM (2016) 787, online: https://ec.europa. $\mathrm{eu} /$ transparency $/ \mathrm{regdoc} /$ ?fuseaction $=1$ ist $\& \mathrm{n}=10 \& \mathrm{adv}=0 \& \operatorname{cote} I \mathrm{~d}=1 \&$ year $=2016 \&$ number $=787 \&$ language $=$ it $(30.05 .2020)$.

${ }^{82} \mathrm{Cfr}$. Comunicazione della Commissione del 11 dicembre 2019 al Parlamento europeo, al Consiglio, al Comitato economico e sociale europeo e al Comitato delle regioni: "Il Green Deal Europeo", $\operatorname{COM}(2019) 640$ def., online: https://eur-lex.europa.eu/legal-content/IT/TXT/?uri=CELEX:52019DC0640 (30.05.2020). 
e scaturenti dalla mobilità urbana, sono specifiche per ogni singola città e comprendono: ${ }^{83}$

a) lo sviluppo di sistemi di trasporto pubblico efficaci, equilibrati, sicuri e protetti, incluso il sistema Mobility-as-a-Service $(\mathrm{MaaS})^{84}$ e altre piattaforme;

b) l'adeguamento all'innovazione e l'accettazione di nuove forme di veicoli (autonomi, collegati, elettrici, di comunità, senza stazionamento);

c) lo sviluppo di linee guida e di strategie per promuovere il rispetto delle norme sulla qualità dell' aria e di altre misure per il miglioramento della qualità di vita;

d) lo sviluppo di partenariati pubblico-privati (PPP) e la cooperazione con istituti scientifici per affrontare questioni di qualità dell'aria, di congestione del traffico e di sostenibilità;

e) la costruzione di un'infrastruttura sostenibile, sia fisica che digitale, per sostenere soluzioni di mobilità innovative del settore pubblico e privato.

Non da ultimo, massima priorità va data all'introduzione di misure per la riduzione e il controllo dell'inquinamento. La relazione speciale 06/2020 della Corte dei conti europea presentata in virtù dell' articolo 287 , paragrafo 4 , secondo comma, del TFUE, rimarca che la mobilità urbana sostenibile è una delle principali sfide che le città dell'UE si trovano ad affrontare, destando non poca preoccupazione in molti cittadini europei. I trasporti stradali rappresentano infatti una delle principali cause dell'inquinamento atmosferico e delle emissioni di gas a effetto serra nelle aree urbane, e i costi della congestione per la società ammontano a circa 270 miliardi di euro all'anno. ${ }^{85}$ Le ripercussioni di tali fenomeni sulla salute degli individui, com'è intuibile, sono estremamente gravi e non possono essere sottovalutate. ${ }^{86}$

\footnotetext{
${ }^{83}$ Cfr. Jon Glasco, Urbane Mobilität: Herausforderungen und Lösungen in Smart Cities (09.10.2019), online: https://hub.beesmart.city/de/smart-city-loesungen/urbane-mobilitaet-herausforderungen-und-loesungen-in-smart-cities (30.05.2020).

${ }^{84}$ Per Mobility-as-a-Service si intende una nuova modalità di pianificare il proprio viaggio urbano. Lo smart citizen non organizza più in prima persona il percorso di viaggio gestendo singolarmente ogni passaggio e ogni tratta. MaaS consente di prenotare in un unico servizio omnicomprensivo tutti i mezzi necessari per il viaggio urbano da un'unica piattaforma, pagando con abbonamento o a forfeit e ottenendo anche suggerimenti su veicoli e percorsi migliori; cfr. Peraphan Jittrapirom/ Valeria Caiati/Anna-Maria Feneri/Shima Ebrahimigharehbaghi/María J. Alonso González/Jishnu Narayan, Mobility as a Service: A Critical Review of Definitions, Assessments of Schemes, and Key Challenges, in: Urban Planning, 2, 2, 2017, pp. 13 ss., DOI: 10.17645/up.v2i2.931.

${ }^{85} \mathrm{Cfr}$. Corte dei Conti europea (a cura di), Relazione speciale 06/2020. Mobilità urbana sostenibile nell'UE: senza l'impegno degli Stati membri non potranno essere apportati miglioramenti sostanziali, Lussemburgo, 2020, p. 6 e passim, online: https://www.eca.europa.eu/it/Pages/DocItem.aspx?did=53246 (30.05.2020).

${ }^{86}$ Secondo una comunicazione della Commissione europea le morti premature nell'UE dovute all'inquinamento provocato dai trasporti sono quasi tre volte superiori a quelle causate dagli incidenti stradali. Inoltre, milioni di europei soffrono di malattie respiratorie e cardiovascolari croniche che sono la immediata conseguenza di tale inquinamento; cfr. Comunicazione della Commissione del 31 maggio 2017 al Parlamento europeo, al Consiglio, al Comitato economico e sociale europeo e al Comitato delle regioni: "L'Europa in Movimento. Un'agenda per una transizione socialmente equa verso una mobilità pulita, competitiva e interconnessa per tutti”, COM (2017)283 def., online: https://eur-lex.europa.eu/legal-content/IT/TXT/?uri=CELEX\%3A52017DC0283 (30.05.2020)
} 
Sempre più spesso, pertanto, alla politica dei trasporti viene richiesto di ispirarsi ai principi di una green mobility. ${ }^{87}$ Anche se questo concetto per molti versi pare eccessivamente enfatizzato dall'industria automobilistica per promuovere i propri veicoli elettrici, è innegabile che oggi quasi tutte le grandi aziende nel settore automobilistico sviluppino e vendano autovetture elettriche.

L'urbanizzazione e la continua dipendenza dalle automobili determinano inevitabilmente problemi di congestione del traffico, emissioni e problemi di sicurezza stradale che richiedono una soluzione politica e urbanistica. Pur riconoscendo le città i benefici derivanti del trasporto pubblico per la riduzione dell'inquinamento e della congestione stradale, gli sforzi compiuti dalle amministrazioni comunali per sfruttare tali vantaggi si trovano spesso in collisione con nuovi modelli imprenditoriali come Uber $^{88}{ }^{8 y f t},{ }^{89} \mathrm{DiDi}^{90}$ e altri servizi di ride-hailing (richiesta di passaggio). La popolarità del ride-hailing ha trasformato il mercato dei trasporti in molte città tanto che in alcuni casi Uber è considerato una minaccia per le città, in quanto idoneo ad aumentare l'inquinamento e la congestione stradale e parallelamente a diminuire il numero dei passeggeri dei mezzi di trasporto pubblici. D'altro canto, per molti utenti - soprattutto nelle periferie delle grandi città - il trasporto pubblico rappresenta tutt'ora un'opzione poco attraente e praticabile se le fermate sono troppo distanti dal loro domicilio o dal posto di lavoro. Si pone quindi il problema del primo e dell'ultimo tratto del trasporto pubblico, a cui il ride-hailing e le altre offerte di sharing potrebbero ovviare, divenendo di sostegno al trasporto pubblico e limitando così la mobilità automobilistica individuale. La sfida consiste dunque proprio nell'integrazione dei mezzi pubblici con servizi di ride-hailing, ridesharing, vehicle-sharing e smart ticketing. ${ }^{91}$

Nonostante l'adozione di valide misure volte a migliorare la sicurezza della mobilità urbana, i morti a causa di incidenti stradali sono in aumento in molte città europee. La densità demografica urbana, combinata ad automobili, automezzi pesanti e mezzi di trasporto pubblico che condividono strade affollate con utenti della strada indifesi (pedoni, ciclisti e motociclisti), rende il compito di garantire una mobilità sicura un'impresa complessa. Il problema della sicurezza è ulteriormente aggravato da abitudini di guida pericolose e da un'infrastruttura inadeguata per i ciclisti e gli utenti della micro-mobilità (motorini, monopattini ecc.).

Inoltre, a causa del crescente utilizzo di tecnologie digitali il settore dei trasporti si trova ad affrontare maggiori rischi di sicurezza informatica. I criminali informatici sono sempre più in grado di attaccare non solo la tecnologia dell'informazione, ma anche la tecnologia operativa che gestisce i sistemi di segnalazione e di controllo

\footnotetext{
${ }^{87} \mathrm{Cfr}$. Andrea Poggio (a cura di), Green mobility. Come cambiare la città e la vita, Milano, 2018.

${ }^{88} \mathrm{Cfr}$. https://www.uber.com/at/en/ (30.05.2020).

${ }^{89} \mathrm{Cfr}$. https://www.lyft.com/ (30.05.2020).

${ }^{90} \mathrm{Cfr}$. https://www.didiglobal.com/ (30.05.2020).

${ }^{91} \mathrm{Cfr}$. Jon Glasco, Urbane Mobilität: Herausforderungen und Lösungen in Smart Cities (09.10.2019), online: https://hub.beesmart.city/de/smart-city-loesungen/urbane-mobilitaetherausforderungen-und-loesungen-in-smart-cities (30.05.2020).
} 
di una città. I cyberattacchi possono colpire le reti di trasporto urbano e causare disfunzioni nei trasporti pubblici, come anche in tutte le vetture autonome. ${ }^{92} \mathrm{La}$ tutela dei diritti fondamentali alla protezione dei dati a carattere personale è anche in questo contesto un tema particolarmente sensibile e sottovalutato. Lo smart citizen, che sarà in ogni momento monitorato, è infatti sottoposto a una continua minaccia di compressione dei propri diritti fondamentali in tema di dati. A tal proposito, basti pensare che alcuni Stati - come ad esempio la Cina ${ }^{93}$ - hanno già iniziato a spiare a tappeto i propri cittadini utilizzando vecchie e nuove tecnologie come spyware nei telefoni cellulari, telecamere per il riconoscimento facciale, wi-fi sniffer ecc. Fintanto che i dati rimangono nella sfera di controllo di enti pubblici obbligati per primi al rispetto, istituzionalmente loro imposto, dei diritti fondamentali, la minaccia di violazione dei diritti fondamentali è ridotta; ben altra cosa è, invece, se questi dati giungono nelle mani di imprese private orientate alla massimizzazione del loro profitto.

Ad oggi il mercato offre una moltitudine di soluzioni per una mobilità urbana intelligente. Solo il futuro saprà dirci tuttavia quali tra queste soluzioni riusciranno effettivamente ad imporsi su un mercato europeo che appare molto conteso, peraltro anche da Stati Uniti ed e Paesi del mondo asiatico. Tra gli esempi ${ }^{94}$ che meritano di essere segnalati osserviamo: Moovel, ${ }^{95}$ una piattaforma innovativa di MaaS, che combina e facilita l'utilizzo di servizi di trasporto multimodali e di shared mobility (mobilità condivisa) ${ }^{96} \mathrm{e}$ consente il pagamento attraverso un' unica interfaccia. Innovactory, ${ }^{97}$ che si è impegnata a rendere più sostenibile il comportamento di viaggio dei suoi utenti con lo sviluppo di TimesUpp ${ }^{98}$ un assistente di viaggio intelligente,

\footnotetext{
${ }^{92} \mathrm{Cfr}$. Ibid.

${ }^{93} \mathrm{Cfr}$. Barbara Calderini, Sorveglianza di massa in Cina, il modello che spaventa l'Occidente, (04.03.2020), online https://www.agendadigitale.eu/sicurezza/privacy/sorveglianza-di-massa-in-cina-cosi-funziona-il-modello-che-spaventa-loccidente/ (30.05.2020). Secondo tale articolo Xue Liang (= occhio di falco) è il nome del programma di videosorveglianza a tappeto del Governo cinese a Pechino presieduto da Xi Jinping. Si tratta di un network di sorveglianza molto complesso, onnipresente e totalmente connesso che comprende progetti di videosorveglianza di massa che incorporano la tecnologia di riconoscimento facciale compreso quello emozionale, software di riconoscimento vocale in grado di identificare gli altoparlanti durante le telefonate; e un programma ampio e invadente di raccolta del DNA.

${ }^{94}$ Cfr. Jon Glasco, Urbane Mobilität: Herausforderungen und Lösungen in Smart Cities (09.10.2019), online: https://hub.beesmart.city/de/smart-city-loesungen/urbane-mobilitaetherausforderungen-und-loesungen-in-smart-cities (30.05.2020).

${ }^{95} \mathrm{Cfr}$. https://www.moovel.com/en (30.05.2020). Questa soluzione intelligente per la mobilità urbana offre una funzione multimodale che riunisce opzioni di trasporto come i mezzi pubblici, i servizi on-demand (su richiesta) e i servizi di car-sharing, bike-sharing e ride hailing. Mediante l'app Moovel i clienti possono prenotare e pagare servizi di mobilità attraverso un conto integrato. ${ }^{96}$ I principali esempi sono il car sharing, bike sharing e scooter sharing. La shared mobility si può estendere però anche a hoverboard, segway, monopattini, monowheel, v. per questi ultimi Decreto del Ministero delle Infrastrutture e dei Trasporti, 4 giugno 2019: "Sperimentazione della circolazione su strada di dispositivi per la micromobilita' elettrica", in. Gazzetta Ufficiale, 12.07.2019, n. 162.

${ }^{97}$ Cfr. https://innovactory.com/ (30.05.2020).

${ }^{98} \mathrm{Cfr}$. https://timesupp.com/ (30.05.2020).
} 
che consiglia il momento migliore e il mezzo di trasporto migliore per giungere alla destinazione prescelta dall'utente, fornendogli informazioni in tempo reale su ingorghi e su altri impedimenti imprevisti. PSIRoads è una soluzione intelligente per la gestione del traffico che fornisce un supporto decisionale attraverso l'intelligenza artificiale. Questa soluzione intelligente di mobilità urbana fornisce servizi di gestione del traffico, come i cambiamenti di fase dei semafori, informazioni sugli utenti del traffico e sui cambiamenti dinamici dell'intensità del traffico. ${ }^{99}$ Parquery, ${ }^{100}$ una soluzione cloud-based di smart-parking, fornisce ai gestori dei parcheggi dati precisi sull'utilizzo e la disponibilità dei parcheggi. ${ }^{101}$ Invero, parte della congestione stradale nelle aree urbane è causato da utenti alla ricerca di un parcheggio.

Sulla micro-mobilità si sta già oggi puntando con successo, investendo risorse, susistemi e flotte di biciclette e monopattini elettrici in sharing. (e-scooter-sharing). eCooltra ${ }^{102}$ è un innovatore europeo nel campo dei monopattini in sharing, che dispone di una flotta di monopattini elettrici operativa in cinque città. Mediante l'app eCooltra gli utenti possono prenotare e sbloccare un monopattino disponibile e pagare solo per i minuti di effettivo utilizzo.

Nel settore del trasporto pubblico locale (TPL) l'informazione trasparente ai passeggeri è ormai d’obbligo, ciò significa in concreto che in un sistema di smart mobility, nel sistema di TPL classico, vi dovrà essere l'installazione di sistemi GSM e GPRS in tutti i veicoli di trasporto della persona, vi dovranno essere le indicazioni elettroniche alle fermate degli autobus, vi dovrà essere un software di pianificazione per i centri di controllo e un sito web con informazioni dinamiche per i passeggeri accessibile in ogni momento a da ogni apparecchio collegabile ad internet.

Vale la pena citare infine il progetto HiReach, ${ }^{103}$ un' attività di ricerca e innovazione finanziata dal programma UE Horizon 2020 (anche Orizzonte 2020), ${ }^{104}$ ha l'obiettivo di trovare soluzioni per migliorare l'accessibilità, il coinvolgimento e l'organizzazione equa della mobilità e comprende: (i) la ricerca di modelli commerciali praticabili per servizi di mobilità convenienti, modulari e ripetibili (trasporto pubblico locale, car-sharing, minibus); (ii) la generazione e la sperimentazione di soluzioni di mobilità di start-up e imprese; (iii) la realizzazione della fattibilità e della scalabilità di nuovi modelli commerciali di mobilità. ${ }^{105}$

\footnotetext{
${ }^{99} \mathrm{Cfr}$. https://www.psi-minesandroads.de/en/home/ (30.05.2020). PSIRoads intende aiutare le autorità preposte al traffico a raggiungere obiettivi strategici, minimizzando le emissioni dei veicoli e riducendo la congestione del traffico in zone residenziali.

${ }^{100} \mathrm{Cfr}$. https://parquery.com/it/ (30.05.2020). Parquery sostiene anche la gestione adattabile dell'illuminazione stradale, la gestione intelligente del traffico e servizi retail (al dettaglio) per facilitare la navigazione in una smart city.

${ }^{101}$ La ditta Urbiotica offre un sistema simile, v., https://www.urbiotica.com//(30.05.2020).

${ }^{102} \mathrm{Cfr}$. https://www.ecooltra.com/it/(30.05.2020).

${ }^{103} \mathrm{Cfr}$. https://hireach-project.eu/ (30.05.2020)

${ }^{104}$ Cfr. https://ec.europa.eu/programmes/horizon2020/ (30.05.2020).

${ }^{105} \mathrm{Cfr}$. Jon Glasco, Urbane Mobilität: Herausforderungen und Lösungen in Smart Cities (09.10.2019), online: https://hub.beesmart.city/de/smart-city-loesungen/urbane-mobilitaetherausforderungen-und-loesungen-in-smart-cities (30.05.2020).
} 
Viste - concludendo - le tendenze innovative e le forze imprenditoriali attive a livello globale nel settore della mobilità urbana, è realistico immaginare uno scenario futuro in cui gli abitanti e i visitatori di una smart city usufruiscano di una vasta gamma di opzioni di mobilità on- demand, convenienti e multimodali, e in cui le automobili convenzionali e le prassi di proprietà sono sostituite da veicoli comuni elettrici e autonomi. Questo potrebbe effettivamente portare ad un calo del numero dei decessi per incidenti stradali, alla riduzione dei tempi di viaggio (vantaggio per i pendolari e per l'economia bloccata da ingorghi stradali), ma anche alla riduzione del devastante fenomeno dell'inquinamento atmosferico. Ad ogni modo, la qualità di una mobilità del futuro nelle città europee - anche per lo spirito che anima l' Unione Europea - verrà commisurata anche in base alla capacità di trovare soluzioni per le persone che non hanno le risorse economiche per disporre di un'automobile, che incontrano difficoltà nel finanziare le tariffe crescenti dei mezzi pubblici e che, a causa dell'età, della disabilità o del luogo di residenza, non hanno accesso ai trasporti pubblici o privati.

Open Access Dieses Kapitel wird unter der Creative Commons Namensnennung 4.0 International Lizenz (http://creativecommons.org/licenses/by/4.0/deed.de) veröffentlicht, welche die Nutzung, Vervielfältigung, Bearbeitung, Verbreitung und Wiedergabe in jeglichem Medium und Format erlaubt, sofern Sie den/die ursprünglichen Autor(en) und die Quelle ordnungsgemäß nennen, einen Link zur Creative Commons Lizenz beifügen und angeben, ob Änderungen vorgenommen wurden.

Die in diesem Kapitel enthaltenen Bilder und sonstiges Drittmaterial unterliegen ebenfalls der genannten Creative Commons Lizenz, sofern sich aus der Abbildungslegende nichts anderes ergibt. Sofern das betreffende Material nicht unter der genannten Creative Commons Lizenz steht und die betreffende Handlung nicht nach gesetzlichen Vorschriften erlaubt ist, ist für die oben aufgeführten Weiterverwendungen des Materials die Einwilligung des jeweiligen Rechteinhabers einzuholen. 